\title{
Pig model gets to the dogma
}

\author{
Paul Gerard Bannon, MD, PhD, ${ }^{\mathrm{a}, \mathrm{b}, \mathrm{c}}$ Raffi Qasabian, MBBS, ${ }^{\mathrm{d}}$ and Michael John Byrom, MBChB, PhD ${ }^{\mathrm{a}, \mathrm{b}}$
}

See related article on pages $413-4$.

Knowledge of the anatomy and physiology of the blood supply to the spinal cord is essential for efforts to preserve spinal cord perfusion during and after open or endovascular repair of descending thoracic and thoracoabdominal aortic aneurysms to prevent spinal cord injury and the catastrophic complication of paraplegia. Historically, such understanding has focused on the longitudinal vascular connections within the spinal canal, namely, the paired posterior spinal arteries and especially the single midline anterior spinal artery and the input to this from the dominant anterior radicular artery, the artery of Adamkiewicz. Therefore, strategies to maintain spinal cord perfusion have relied on preserving important segmental arterial supply to these connections, including the artery of Adamkiewicz, and maintaining perfusion pressure through attention to systemic arterial pressure supplemented by judicious drainage of cerebrospinal fluid.

More recently, Etz and colleagues ${ }^{1}$ expanded this understanding by undertaking detailed resin casts of the spinal collateral network in juvenile Yorkshire pigs. In this work, the intraspinal collateral system indicated earlier was shown to comprise not only longitudinal channels, such as the anterior spinal artery, but also a series of epidural arcades at each vertebral segment forming a longitudinal chain that connects extensively to the anterior spinal artery and anastomotic networks on either side. However, this intraspinal system is supplemented by a larger extrathecal paraspinous collateral network, mostly arterioles and capillaries, with a volume 25 times that of the intraspinal system. The paraspinous network is supplied by dorsal branches of the segmental arteries and comprises vessels to the paravertebral tissues lying along the dorsal processes of the vertebral

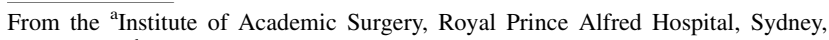
Australia; ${ }^{b}$ Department of Cardiothoracic Surgery, Royal Prince Alfred Hospital, Sydney, Australia; ${ }^{\mathrm{c}}$ University of Sydney, Sydney, Australia; and ${ }^{\mathrm{d}}$ Department of Vascular Surgery, Royal Prince Alfred Hospital, Sydney, Australia.

Disclosures: Authors have nothing to disclose with regard to commercial support.

Received for publication April 2, 2015; accepted for publication April 3, 2015; available ahead of print June 26, 2015.

Address for reprints: Paul Gerard Bannon, MD, PhD, Department of Cardiothoracic

Surgery, Royal Prince Alfred Hospital, Missenden Road, NSW 2050, Australia

(E-mail: pgbannon@gmail.com).

J Thorac Cardiovasc Surg 2015;150:415-6

$0022-5223 / \$ 36.00$

Copyright (C) 2015 by The American Association for Thoracic Surgery

http://dx.doi.org/10.1016/j.jtcvs.2015.04.059
}

bodies and especially vessels to the paraspinous muscles, including the iliopsoas anteriorly and the erector spinae posteriorly. Like the intraspinal network, these paraspinous vessels are interconnected with multiple longitudinal anastomoses along the vertebral column. Taken together, these networks suggest a longitudinally continuous system fed by the segmental arteries and input from subclavian and hypogastric arteries whereby particular inputs from significant segmental arteries may be less important than previously proposed, and the preservation of more distal inputs to the collateral system and prevention of steal from the spinal cord circulation by early postoperative paravertebral muscle activity may be critical.

In the report by Kari and colleagues, ${ }^{2}$ a large animal model is described for investigation of the intraspinal collateral system only through percutaneous ethanol ablation of the dorsal segmental arteries (T3-L5) supplying the paraspinous collateral network. Six juvenile pigs underwent blind bilateral percutaneous ablation under general anesthesia, with the effect confirmed by pre- and postinterventional computed tomography and magnetic resonance angiography, and histologic examination after the animals were killed at 7 days. All animals recorded mild temporary reduction in hindlimb motor function that returned to normal by 2 to 4 days postintervention except for 1 animal that showed permanent paraparesis and was killed on day 3 . While supporting the porcine model for spinal cord blood supply and its relevance to the human clinical situation, Etz and colleagues ${ }^{1}$ note the presence of 13 thoracic vertebrae in the pig rather than 12 in the human and the potential impact of aortic disease in older adults on the collateral circulation to the spinal cord. Nevertheless in the current small study, Kari and colleagues $^{2}$ successfully isolate spinal collateral flow to that delivered by the intraspinal network with the aim of better characterizing the details and importance of this system. The utility of this model will ultimately depend on its use in the formulation of strategies to preserve spinal cord perfusion and reduce neurologic injury in the conduct of aortic replacement or endovascular exclusion. It is equally essential to develop models that can simulate the removal of the segmental arterial supply to the intraspinal system and leave the paraspinous network intact, simulating the effect of operative intervention to the descending thoracic and abdominal aorta. 


\section{References}

1. Etz CD, Kari FA, Mueller CS, Silovitz D, Brenner RM, Lin HM, et al. The collateral network concept: a reassessment of the anatomy of spinal cord perfusion. J Thorac Cardiovasc Surg. 2011;141:1020-8.
2. Kari FA, Siepe M, Beyersdorf F. Aortic surgical model to study isolated intraspinal collateralization. J Thorac Cardiovasc Surg. 2015;150:413-4. 